\title{
Automation, consolidation, and integration in autoimmune diagnostics
}

\author{
Renato Tozzoli ${ }^{1} \cdot$ Federica D'Aurizio $^{1} \cdot{\text { Danilo } \text { Villalta }^{2} \cdot \text { Nicola Bizzaro }^{3}}^{3}$
}

Received: 15 April 2015/Accepted: 1 June 2015/Published online: 3 July 2015

(C) The Author(s) 2015

\begin{abstract}
Over the past two decades, we have witnessed an extraordinary change in autoimmune diagnostics, characterized by the progressive evolution of analytical technologies, the availability of new tests, and the explosive growth of molecular biology and proteomics. Aside from these huge improvements, organizational changes have also occurred which brought about a more modern vision of the autoimmune laboratory. The introduction of automation (for harmonization of testing, reduction of human error, reduction of handling steps, increase of productivity, decrease of turnaround time, improvement of safety), consolidation (combining different analytical technologies or strategies on one instrument or on one group of connected instruments) and integration (linking analytical instruments or group of instruments with preand post-analytical devices) opened a new era in immunodiagnostics. In this article, we review the most important changes that have occurred in autoimmune diagnostics and present some models related to the introduction of automation in the autoimmunology laboratory, such as automated indirect immunofluorescence and changes in the two-step strategy for detection of autoantibodies; automated monoplex immunoassays and reduction of
\end{abstract}

Renato Tozzoli

renato.tozzoli@aopn.sanita.fvg.it

1 Department of Laboratory Medicine, Laboratory of Clinical Pathology, S. Maria degli Angeli Hospital, Via Montereale, 24, 33170 Pordenone, Italy

2 Allergology-Clinical Immunology, Department of Laboratory Medicine, S. Maria degli Angeli Hospital, Pordenone, Italy

3 Laboratory of Clinical Pathology, San Antonio Hospital, Tolmezzo, Italy turnaround time; and automated multiplex immunoassays for autoantibody profiling.

Keywords Automated immunofluorescence .

Automated monoplex immunoassay .

Automated multiplex immunoassay .

Two-step diagnostic strategy - Turnaround time .

Autoantibody profiling

\section{Introduction}

Over the past 25 years, autoimmune diagnostics has gone through an evolutionary period, which seems not yet ended, as clinical and basic knowledge in the field of autoimmunity is still growing. From year to year, important achievements in pathophysiology, development of new diagnostic technologies and advances in therapy of autoimmune diseases (AIDs) have been steadily added. The engine of this evolutionary period is the improvement in AIDs diagnostics, which has allowed an increasing number of recognized cases of individuals (3-8\% of the population; $80 \%$ women) suffering from one or more AIDs $[1,2]$. The main recent cornerstones in this field have been: (a) The discovery of new autoantibody-autoantigen systems (both systemic and organ-specific); (b) Recognition that autoantibodies may have predictive, pathogenic, or protective roles; (c) The availability of new diagnostic technologies (monoplex and multiplex immunoassays-IMA), and (d) Changes in organizational processes (integration and automation) [3-6].

We could now wonder if such events could have been foreseen at the beginning of the era of modern autoimmune diagnostics. The answer to this question lies in the expected changes in clinical immunology laboratories as dictated in the early 1990s. Twenty years ago, Nakamura 
and Bylund [7], during the 17th AO Beckman conference, identified the primary focus of the laboratory of clinical immunology and defined the main influencing factors of future changes: governmental regulations, healthcare economics, advances in technology and advances in development and use of therapeutic agents. At the same time, Hamilton [8], looking at the clinical immunology laboratory of the future, emphasized the importance of standardization of methods and development of 'universal' automated immunoanalyzers. Two years later, Normansell [9], at the 8th annual meeting of the American Association of Medical Laboratory Immunologists, foresaw the progressive unification of academic specialty laboratories (chemistry, hematology, immunology, etc.) in a unique general laboratory (consolidation).

Most of these predictions have been achieved, and especially automation and consolidation paved the way to the modern laboratory $[10,11]$. During the past decade, we and others [12-16] have described the progressive evolution of analytical methods and technologies, signaling the extraordinary dynamic development of autoimmune diagnostics resulting from the availability of new tests, diffusion of improved immunoassay methods and instruments, and the explosive growth of molecular biology and proteomic medicine.

To meet both clinical need and growing demand for autoantibody testing, automation of autoimmune diagnostics has invaded the laboratory as an extension of the general technological improvement already achieved in almost all other diagnostic areas of the clinical laboratory [17, 18]. Currently, all stages of the analytical procedure for detection and quantification of autoantibodies are automated. The third generation of laboratory systems now encompasses most of the analytical steps of the laboratory workflow, enabling the clinical pathologists (autoimmunologists in this case) to focus on 'value-added' work, such as result validation and production of narrative reports for clinical interpretation [4].

The two key concepts of third generation systems are 'consolidation' (i.e., combining different analytical technologies or strategies on one instrument or on one group of connected instruments) and 'integration' (linking analytical instruments or group of instruments with pre- and post-analytical devices).

As predicted, in many cases this approach has brought about the reunification of diagnostics in the central general laboratory. It is now customary to manage the flow of highly demanded autoantibody tests (thyroperoxidase, thyroglobulin, tissue transglutaminase, citrullinated peptide autoantibodies) in 'human-less' robotic platforms, characterized by total laboratory automation (TLA) which includes pre-analytical, analytical, and post-analytical operations [19]. The rationale in favor of this approach lies in the advantage of placing as many assays on a single analyzer, rather than maintaining two or more analyzers, because each instrument requires separate quality control (QC), preventive maintenance, record keeping, etc. (Figure 1). TLA combines a large variety of processes, including accessioning and sorting specimens, decapping tubes, centrifugation, aliquoting, delivery to analyzers, recapping tubes, and storage and archiving of samples. The prototype of instruments used in the autoimmunology laboratories is IMA platforms [4-6].

The advantages of TLA include harmonization of testing to improve patient care [20], reduction of human error, reduction of handling steps, increase of productivity, decrease and standardization of turnaround time (TAT), improvement of safety, use of state of the art informatics (i.e., interface with laboratory information systems-LIS and health information systems; middleware to bridge the analyzers and LIS). Most, if not all, the obstacles to automation as defined by Tomar as much as 15 years ago [10] are now completely removed.

In the next sections of this article, we present some models related to the introduction of automation in the autoimmunology laboratory: a. automated indirect immunofluorescence (IIF) and the changes in the two-step strategy for detection of autoantibodies, b. automated monoplex IMAs and the reduction of TAT, and c. automated multiplex IMAs and autoantibody profiling.

\section{Automated indirect immunofluorescence and changes in the two-step strategy for autoantibody detection}

Recently, indirect immunofluorescence (IIF) automation for reading and interpretation of ANA and other autoantibodies was developed, supported by the American College

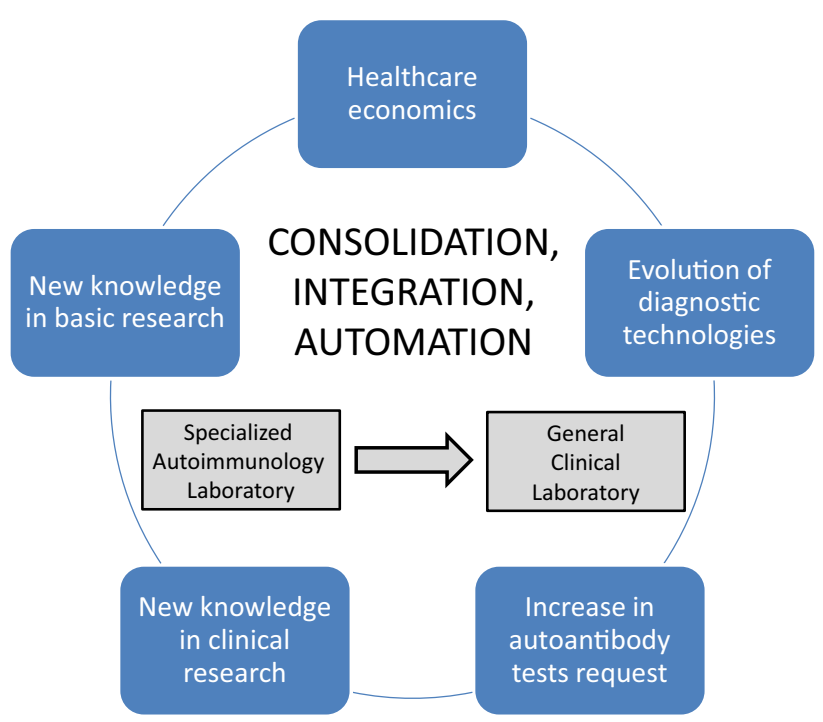

Fig. 1 Evolution of clinical immunology laboratories 
of Rheumatology recommendation that IIF is the reference method for anti-nuclear-cytoplasmic antibodies (NCA or ANA) [21, 22], anti-neutrophil cytoplasmic antibodies (ANCA) [23], and anti-dsDNA antibodies (DNAAb). At present, at least 6 automatic systems for the detection of these autoantibodies are available [4, 24, 25] (Table 1). These systems allow for automated classification of samples, with a high efficiency in discriminating between positive and negative ANA and an acceptable correlation with manual microscope reading [4, 26, 27], automated quantification of autoantibodies [28, 29], and implementation of a quantitative internal QC system [30].

These features allow a different approach to the diagnostic strategy for the detection of NCA-ANA, ANCA, and DNAAb, based on a two-step algorithm: the first stage, the screening of positive/negative and positive samples selection; the second stage, identification of specific antibodies for confirmation of screening results and classification of autoimmune diseases [33, 34].

This flow of information in autoimmune diagnostics has long been conducted with the strategy of reflective testing. That is, the morphologist identifies positive samples using the IIF method and, on the basis of the fluorescence pattern, decides which autoantibodies are to be detected by IMA methods. Moreover, if necessary, reflective testing provides for the addition of new tests beyond the test originally requested and/or production of comments to aid in the correct interpretation of results by the clinical autoimmunologist. Reflective testing can be now replaced with reflex testing, in which a predetermined test protocol is automatically completed [35]: the integration and the use of specific health information technologies allow now the automated application of rules and the computerized addition of specific tests with high efficiency and effectiveness (Table 2), reducing the need of morphologists and the TAT for clinical applications.

Automation of IIF and the use of specific health information technology systems applied to the autoimmunology laboratory, besides allowing for the automated classification of negative/positive results, permit also the practice of pathology at a distance (telepathology, or digital microscopy, or whole slide imaging). Telepathology allows the remote interpretation of fluorescence patterns (telediagnosis), second opinions or consultations (teleconsultation), quality assurance, education, teaching, self-study and research (teleeducation) [31]. Telepathology has been successfully applied to anatomic pathology, hematology, and microbiology; its use in biomedicine is rapidly evolving [32].

\section{Monoplex immunoassays and the reduction of turnaround time}

Automated IIF and third-generation monoplex IMAs allow the rapid detection of autoantibodies in terms of minutes or hours, instead of days or weeks as required by manual IIF and IMA methods. In the past, fast autoantibody results did not represent a main goal for clinicians and autoimmunologists,
Table 1 Currently available automated IIF platforms ( $H$ homogeneous, $S$ speckled, $N$ nucleolar, $C$ centromere, $N D$ nuclear dots, $N M$ nuclear membrane, $C y$ cytoplasmic)

Table 2 Time evolution of two-step strategy for NCA/ANA detection/measurement

\begin{tabular}{llll}
\hline System & Screening neg/pos & Patterns (no; type) & Company \\
\hline Aklides & Yes & 6-H, S, N, C, ND, Cy & Medipan, Germany \\
EUROPattern & Yes & 7-H, S, N, C, ND, NM, Cy & Euroimmun, Germany \\
Zenit G-Sight & Yes & 5-H, S, N, C, M & A. Menarini Diagnostics, Italy \\
NOVA view & Yes & 5-H, S, N, C, ND & Instrumentation Laboratories, Spain \\
Helios & Yes & - & Aesku Diagnostics, Germany \\
Image navigator & Yes & - & ImmunoConcepts, USA \\
\hline
\end{tabular}

\begin{tabular}{|c|c|c|}
\hline Era & Years & Methods \\
\hline Manual ANA IIF-reflective adding on-manual ENA & $1970-2000$ & $\begin{array}{l}\text { Manual IIF } \\
\text { Manual monoplex methods } \\
\text { Low automated monoplex methods }\end{array}$ \\
\hline Manual ANA IIF-reflective adding on-automated ENA & 2000-2014 & $\begin{array}{l}\text { Manual IIF } \\
\text { Full automated monoplex methods } \\
\text { Low automated multiplex methods }\end{array}$ \\
\hline Automated ANA IIF-reflex adding on-automated ENA & $2015-\ldots$ & $\begin{array}{l}\text { Automated IIF } \\
\text { Full automated monoplex methods } \\
\text { Full automated multiplex methods }\end{array}$ \\
\hline
\end{tabular}

For the terminology see text 
which in many cases utilized longer turnaround times for repetition of positive tests or for second opinions. In current practice, however, life-threatening autoimmune conditions or autoimmune diseases associated with rapidly progressive loss of organ function have emerged, requiring a short response time to allow rapid etiological and differential diagnosis. This, in turn, will enable the start of immunosuppressive or other specific therapies as soon as possible. This is the case, for example, for ANCA-associated small-vessel vasculitides or Goodpasture' syndrome [36], or for patients with acute cardiovascular symptoms (thyroid storm, atrial fibrillation, supraventricular tachycardia, etc.) in undiagnosed TSH-receptor antibody-dependent Graves' disease [37, 38]. Another condition involves patients affected by catastrophic antiphospholipid syndrome with concurrent thrombotic and hemorrhagic manifestations [39]. In these clinical settings such as may occur in intensive-care units, the rapid availability of autoantibody tests is a great opportunity for early diagnosis and treatment to save a patient's life [40].

Furthermore, the availability of automated analyzers with reduced assay times (within $120 \mathrm{~min}$ ) as well of manual pointof-care systems [41] enables real-time antibody measurement in the same day of the request or even in stat mode, avoiding delay and improving compliance towards diagnosis, differential diagnosis and therapy monitoring in the clinical conditions listed above [41]. This also responds to the increasing need for faster diagnosis owing to shorter period of hospitalization. A partial list of automated platforms, with their assay times and throughput, is shown in Table 3.

\section{Automated multiplex IMAs and autoantibody profiling}

Multiplex proteomic technology is considered to be an optimal solution for the simultaneous detection of different autoantibodies related to AIDs. Some of these immunoassays (planar and non-planar microarrays) may contribute to overcoming some drawbacks of the monoplex immunoassays (time expenditure, costs, lack of harmonization, volume of reagents and samples, turnaround time, etc.) [5].

The autoantibody profiling of AIDs patients provided by these systems may be useful for following the concentration of specific autoantibodies, which may display different trends over time, both for diagnostic and prognostic purposes. This is the case, for instance, of celiac disease and anti-phospholipid syndrome which are characterized by the presence of multiple autoantibodies of different isotypes. Nowadays, multiplexed technology has achieved high analytical accuracy and provides results comparable to, if not superior to, the manual and automated monoplex technologies [42, 43].

\section{The autoimmunology laboratory of the near future}

The referred changes have altered the scenario and strongly characterized the evolution of the immunology laboratory. But what implications do these new arrangements have for the future?

Avoiding the Ulysses syndrome for the patients Because the results of antibody tests can be available in a few hours, it can be expected that clinical reasoning will occur much faster than now and will involve not only the rheumatologist but also many other specialists and even general practitioners. Signs of this are already visible [44]. If well organized and managed, this change could have positive effects-namely, to ensure greater efficiency of the diagnostic filter, with the ability to identify during their first clinical visit those patients who need a specialist consultation. The advantages for patients, doctors and the health system overall are obvious (Table 4).

Reducing analytical variability of autoantibody tests Another prediction is that measurement of the

Table 3 Automated monoplex IMA platforms

\begin{tabular}{llccc}
\hline $\begin{array}{l}\text { Platform } \\
\text { family }\end{array}$ & Manufacturer & $\begin{array}{l}\text { Autoantibodies detected } \\
(\text { no. })\end{array}$ & $\begin{array}{l}\text { Total assay time } \\
\text { (min) }\end{array}$ & $\begin{array}{l}\text { Throughput (tests per } \\
\text { hour) }\end{array}$ \\
\hline Centaur & $\begin{array}{l}\text { Siemens Healthcare Diagnostics, } \\
\text { Germany } \\
\text { Immulite }\end{array}$ & 3 & 29 & 240 \\
& $\begin{array}{l}\text { Siemens Healthcare Diagnostics, } \\
\text { Germany }\end{array}$ & 4 & 77 & 200 \\
Maglumi & SNIBE Diagnostic, China & 8 & 35 & 180 \\
Cobas & Roche, Germany & 3 & 18 & 170 \\
Architect & Abbott, USA & 4 & 29 & 100 \\
Kryptor & Brahms, Germany & 3 & 19 & 60 \\
BioFlash & Instrumentation Laboratory, Spain & 32 & 30 & 60 \\
Phadia & Thermofisher, Sweden & 47 & 110 & 60
\end{tabular}


Table 4 Automated multiplex IMA platforms

\begin{tabular}{lll}
\hline Instrument & Company & Autoantibody menu (no.) \\
\hline FIDIS & Theradiag, France & 25 \\
Athena Multi-Lyte & Zeus, USA & 19 \\
TruePlex & Origene, USA & 10 \\
Bioplex 2200 & Bio-Rad, USA & 28 \\
Microzyme & ImmunoConcepts, USA & 10 \\
Ig-Plex & SQI Diagnostics, Canada & 22 \\
\hline
\end{tabular}

concentration of autoantibodies, which is still poorly standardized, will become more and more accurate. Subjective methods and semi-quantitative techniques will be replaced by more objective assays and with greater precision, reducing the analytical variability to levels similar to those of other immunoassays. ELISA methods will disappear and blot methods will evolve to provide quantitative results. Measurement of therapeutic antibody levels for follow-up and customization of treatments will become increasingly popular. Costs will drop and autoimmune diagnostics will become part of the clinical profile for early and differential diagnosis.

In conclusion, 20 years after the statements of the prophets about the changes occurring in the management and organization of the clinical immunology laboratory, automation, integration and consolidation allow now for the alignment of the laboratory of autoimmune diseases to other specialized sectors within the general clinical laboratory. This improvement will enable greater harmonization of laboratory autoimmune tests, greater accuracy of test results, and a better outcome for patients with autoimmune diseases.

Conflict of interest The authors have no conflict of interest.

Informed consent For this type of study formal consent is not required.

Ethical approval This article does not contain any studies with human participants performed by any of the authors.

\section{References}

1. Hayter SM, Cook MC (2012) Updated assessment of the prevalence, spectrum and case definition of autoimmune disease. Autoimmun Rev 11:754-765

2. Tozzoli R, Sorrentino MC, Bizzaro N (2013) Detecting multiple autoantibodies to diagnose autoimmune comorbidity (multiple autoimmune syndromes and overlap syndromes): a challenge for the autoimmunologist. Immunol Res 56:425-431

3. Damoiseaux J, Andrade LE, Fritzler MJ, Shoenfeld Y (2015) Autoantibodies 2015: From diagnostic biomarkers toward prediction, prognosis and prevention. Autoimmun Rev (epub ahead of print)
4. Bizzaro N, Tozzoli R, Villalta D (2015) Autoimmune diagnostics: the technology, the strategy and the clinical governance. Immunol Res 61:126-134

5. Tozzoli R, Bonaguri C, Melegari A, Antico A, Bassetti D, Bizzaro N (2013) Current state of diagnostic technologies in the autoimmunology laboratory. Clin Chem Lab Med 51:129-138

6. Tozzoli R, Bizzaro N (2012) The clinical autoimmunologist and laboratory autoimmunologist: the two sides of the coin. Autoimmun Rev 11:766-770

7. Nakamura RM, Bylund DJ (1994) Factors influencing changes in the clinical immunology laboratory. Clin Chem 40:2193-2204

8. Hamilton RG (1994) The clinical immunology laboratory of the future. Clin Chem 40:2186-2192

9. Normansell DE (1996) The Erwin Neter Memorial lecture: looking to the future of medical immunology laboratory, a personal view. Clin Diagn Lab Immunol 3:369-370

10. Tomar R (1999) Total laboratory automation and diagnostic immunology. Clin Diagn Lab Immunol 6:293-294

11. Bock JL (2000) The new era of automated immunoassay. Am J Clin Pathol 113:628-646

12. Wu AHB (2006) A selected history and future of immunoassay development and applications in clinical chemistry. Clin Chim Acta 369:119-124

13. Fritzler MJ (2006) Advances and applications of multiplexed diagnostic technologies in autoimmune diseases. Lupus 15:422427

14. Gonzalez-Buitrago JM, Gonzalez C (2006) Present and future of the autoimmunity laboratory. Clin Chim Acta 365:50-57

15. Villalta D, Tozzoli R, Tonutti E, Bizzaro N (2007) The laboratory approach to the diagnosis of autoimmune diseases: is it time to change? Autoimmun Rev 6:359-365

16. Tozzoli R (2007) Recent advances in diagnostic technologies and their impact in autoimmune diseases. Autoimmun Rev 6:334-340

17. Armbruster DA, Overcash DR, Reyes J (2014) Clinical chemistry laboratory automation in the 21st century-Amat Victoria curam (victory loves careful preparation). Clin Biochem Rev 35:143153

18. Hoffmann GE (1998) Concepts for the third generation of laboratory systems. Clin Chim Acta 278:203-216

19. Hawker CD (2007) Laboratory automation: total and subtotal. Clin Lab Med 27:749-770

20. Plebani M (2013) Harmonization in laboratory medicine: the complete picture. Clin Chem Lab Med 51:741-745

21. Tozzoli R, Bizzaro N, Tonutti E, Villalta D, Bassetti D, Manoni F et al (2002) Guidelines for the laboratory use of autoantibody tests in the diagnosis and monitoring of autoimmune rheumatic diseases. Am J Clin Pathol 117:316-324

22. Agmon-Levin N, Damoiseaux J, Kallenberg C, Sack U, Witte T, Herold $M$ et al (2014) International recommendations for the assessment of autoantibodies to celluar antigens referred to as anti-nuclear antibodies. Ann Rheum Dis 73:17-23 
23. Csernok E, Jolle JU (2010) Twenty-eight years with antineutrophil cytoplasmic antibodies (ANCA): how to test for ANCAevidence-based immunology? Autoimmun Highlights 1:39-43

24. Tozzoli R, Antico A, Porcelli B, Bassetti D (2012) Automation in indirect immunofluorescence testing: a new step in the evolution of the autoimmunology laboratory. Autoimmun Highlights 3:5965

25. Bizzaro N, Antico A, Platzgummer S, Tonutti E, Bassetti D, Pesente F et al (2013) Automated antinuclear immunofluorescence antibody screening: a comparative study of six computeraided diagnostic systems. Autoimmun Rev 13:292-298

26. Buzzulini F, Rigon A, Soda P, Onofri L, Infantino M, Arcarese L et al (2014) The classification of Crithidia luciliae immunofluorescence test (CLIFT) using a novel automated system. Arthritis Res Ther 16:R71

27. Sowa M, Grossmann K, Knutter I, Hiemann R, Rober N, Anderer $\mathrm{U}$ et al (2014) Simultaneous automated screening and confirmatory testing for vasculitis-specific ANCA. PLoS One 9:e107743

28. Bertin D, Jourde-Chiche N, Bongrand P, Bardin N (2013) Original approach for automated quantification of antinuclear autoantibodies by indirect immunofluorescence. Clin Dev Immunol 2013:182172

29. Peng X, Tang J, Wu Y, Yang B, Hu J (2014) Novel method for ANA quantitation using IIF imaging system. J Immunol Methods 404:52-58

30. Maenhout TM, Bonroy C, Verfaillie C, Stove V, Devreese K (2014) Automated indirect immunofluorescence microscopy enables the implementation of a quantitative internal quality control system for anti-nuclear antibody (ANA) analysis. Clin Chem Lab Med 52:989-998

31. Pantanowitz L, Dickinson K, Evans AJ, Hassell LA, Hendricks WH, Lennerz JK et al (2014) American Telemedicine Association clinical guidelines for telepathology. J Pathol Inform 5:39

32. Thrall M, Pantanowits L, Khalbuss W (2011) Telecytology: clinical applications, current challenges, and future benefits. J Pathol Inform 2:51

33. Wiik AS, Bizzaro N (2010) Missing links in high quality diagnostics of inflammatory systemic rheumatic diseases. It is all about the patient! Autoimmun Highlights 3:35-49
34. Damoiseaux JGMC, Cohen Terwaert JW (2006) Fron ANA to ENA: how to proceed? Autoimmun Rev 5:10-17

35. Verboeket-van WPHG, de Venne KM, Aakre Watine J, Oosterhuis WP (2012) Reflective testing: adding value to laboratory testing. Clin Chem Lab Med 50:1249-1252

36. De Joode AAE, Roozendal C, van der Leij MJ, Bungener LB, Sanders JSF, Stegeman CA (2014) Performance of two strategy for urgent ANCA and anti-GBM analysis in vasculitis. Eur $\mathrm{J}$ Intern Med 25:182-186

37. Vaidya B, Pearce SHS (2014) Diagnosis and management of thyrotoxicosis. J Brit Med 349:g5128

38. Lynch MJ, Woodford NWF (2014) Sudden unexpected death in the setting of undiagnosed Graves' disease. Forensic Sci Med Pathol 10:452-456

39. Quintero OL, Rojas-Villarraga A, Mantilla RD, Anaya J-M (2013) Autoimmune diseases in the intensive care unit. An update. Autoimmun Rev 12:380-395

40. Rangel ML, Alghamdi I, Contreras G, Harrington T, Thomas DB, Barisoni L et al (2013) Catastrophic antiphospholipid syndrome with concurrent thrombotic and hemorrhagic manifestations. Lupus 22:854-864

41. Kostantinov KN, Tzamaloukas A, Rubin RL (2013) Detection of autoantibodies in a point-of-care rheumatology setting. Autoimmun Highlights 4:55-61

42. Tozzoli R, Villalta D (2014) Autoantibody profiling of patients with antiphospholipid syndrome using an automated multiplexed immunoassay system. Autoimmun Rev 13:59-63

43. Holding S, Wilson F, Spradbery D (2014) Clinical evaluation of the Bioplex 2200 celiac IgA and IgG kits-A novel multiplex screen incorporating an integral check for IgA deficiency. J Immunol Methods 405:29-34

44. Martin L, Steber WA, Lupton TL, Mahler M, Fitch CM, McMillan JD, et al (2015) Clinical and serological analysis of patients with positive anticyclic citrullinated peptide antibodies referred through a rheumatology central triage system. J Rheumatol (Epub ahead of print) PMID: 25641884 